\title{
¿ZEUXIS O VELÁZQUEZ? LA REIVINIICACIÓN NACIONALISTA EN LA DEFINICIÓN DEL PRIMER NEOCLASICISMO ESPAÑOL
}

\author{
por \\ ANDRÉS UBEDA DE LOS coBOS \\ Centro de Estudios Históricos, C.S.I.C.
}

RESUMEN: Ante las criticas de autores extranjeros, los españoles de finales del siglo XVII sintieron la necesidad de comenzar una reivindicación del pasado español en clave nacionalista. En este debate de carácter general, la historia del arte se convirtio en un argumento de primera magnitud, cuya consecuencia más importante fue la de elevar a Velázquez y a Murillo a la categorfa de paradigmas intemporales.

Palabras clave. Hilstoria del arte. Siglo xvin. Nacionalismo. Velázquez.

ABSTRACT: Reacting to foreign criticism, in the late 18th century, Spaniards felt a need to revindicate the Spanish past with a nationalistic discourse. Within this context history of ant became an important ground for debate. As a result, Velazquez and Murillo were raised to the status of timeless paradigms.

KEY WORDS: History of Art. Eighteenth Century. Nationalism. Velazquez.

El presente trabajo pretende introducir en el estudio del arte del siglo xVIII, particularmente en sus últimos afios, un concepto como es el de patria o patriotismo que habitualmente no se considera en exceso a la hora de llevar a cabo un análisis en esta disciplina. La hipótesis que se apunta es que en estos años el arte comenzó a funcionar en la época indicada con una fuerza hasta entonces desconocida, en lo que hoy calificaríamos como la definición de las características específicas del proyecto nacional español, entonces en construcción. En otras palabras, el desarrollo cultural comenzo a ser entendido como el termómetro del «genio y aplicación de las Naciones» 1.

1 SEMPERE Y GUARINOS, Juan, Reflexiones sobre el buen gusto en las ciencias y en las artes. Traducción libre de las que escribió en italiano Luis Antonio Muratori. Con un discurso sobre el gusto actual de los españoles en literatura, Madrid, Antonio de Sancha, MDCCLXXXII [1782], pág. 16. 
Los estímulos inmediatos que desencadenaron todo este proceso en el terreno específico de las bellas artes fueron, fundamentalmente, de dos tipos distintos: el artículo España de Masson de Morvilliers, en l'Encyclopédie Métodique y, dentro del terreno específico de las bellas artes, el tratamiento otorgado a la escuela española en las Obras del pintor bohemio Antonio Rafael Mengs. En este trabajo pretendo abordar el impacto del primero de los dos asuntos citados y la responsabilidad que puede atribuirse al conocimiento y defensa de las artes -fundamentalmente de la pintura - en la reivindicación de la nación española, frente a los ataques provenientes del exterior.

$\mathrm{El}$ asunto tratado tiene que ver con uno de los grandes mitos de la historia de España: la leyenda negra. Combatir su perverso efecto constituyo, desde su propio origen en el siglo XVI, uno de los afanes intelectuales defendidos con más ímpetu y sus negativos efectos en la imagen que Europa tenía de la Península, llegaron, con salud de hierro, al ilustrado siglo xvin. En efecto, a medida que se fue afirmando una visión del continente europeo como un territorio próspero, cuya industria y comercio parecían crecer de forma ilimitada, confirmando, con ello, la propia conciencia extendida en la época de vivir en un momento de orgulloso progreso; frente a todo esto, un cierto número de mitos antiespañoles se multiplicaron y encontraron su confirmación. Entre ellos, se consolidó una visión de España como el finis terrae de Occidente, presentada invariablemente como el pais de la miseria y la incuria, de la Inquisición, de los prejuicios aristocráticos, la responsable de la Conquista de América sangrienta e injusta, del absurdo rechazo de las denominadas con evidente desdén artes mecanicas, donde de forma incomprensible se denigraban todas las actividades auténticamente productivas de un pais, donde la propiedad de la tierra recaía en manos de las ordenes religiosas... imagen que se encargaron de propagar los abundantes viajeros extranjeros que visitaron este pais ${ }^{2}$. Pensadores franceses y de otros paises de primerísima fila escribieron en estos mismos términos: Montesquieu en sus Lettres persanes, Boyer d'Argens en las Lettres juives, Voltaire en sus obras históricas, Raynal, Diderot, ninguno de ellos inventó nada nuevo con sus críticas a los habitantes de España. Confirmaban, simplemente, una visión que se aceptaba como cierta sin necesidad de justificar sus descalificantes afirmaciones.

Sin embargo, no fue ninguno de estos ilustres pensadores, sino el insignificante publicista Nicolas Masson de Morvilliers ${ }^{3}$, el que provocó una profunda sacudida en la conciencia intelectual española del siglo XVII. Masson

2 En los párrafos siguientes asumo las conclusiones obtenidas por LOPEZ, François, Juan Pablo Forner et la crise de la conscience espagnole au xVIr siècle, Burdeos, Institut d'Études Iberiques et Ibéro-américaines de l'Université, 1976, sobre todo en el capítulo IV, «Forner, apologiste de l'Espagne», págs. 317-436. Vid. también de este mismo autor «Rasgos peculiares de la Ilustración en España), en Mayans y la llustración. Simposio Internacional en el Bicentenario de la muerte de Gregorio Mayans, Valencia, 1981, vol. II, págs. 670 y 671.

3 López, F., lo caracteriza como: «Géographe d'occasion, publiciste sans talent, et de plus fort ignorant des choses de I'Espagne, Nicolas Masson fut, on veut le croire, un des plus médiocres collaborateurs de l'Encyclopédie méthodique. Juan Pablo Forner..., pág. 351.

Hispania, LVII, núm. 192 (1996) 51-62 
fue el autor en 1784 del artículo Espagne en el tomo I, Geographie Moderne, la nueva Encyclopédie Métodique, en el que se hacía la siguiente pregunta: «Que doit-on à l'Espagne? Et depuis deux siècles, depuis quatre, depuis dix, qu'a-telle fait pour l'Europe?». El artículo se dedica a contestar esta retórica cuestión en unos términos altamente descalificantes para España, pais «où il faut demander la permission de penser». El caso concreto de las bellas artes quedó también reflejado en unos términos que confirman plenamente la generalizada opinión de que Masson no era la persona más adecuada para abordar esta obra. Así, a propósito de los principales pintores españoles, señala que son "Velasquez, Murillo, Fançois Guirro, Pierre Cuquet, Jean Arnau, François Gassen, tous natifs de Barcelone». Con estos datos, resulta comprensible la indignación de los españoles.

La apología de España fue asumida como una cuestión de estado por parte del conde de Floridablanca, que sin llegar nunca a ser su promotor, utilizó para ello plumas muy autorizadas como la de Antonio Cavanilles o Juan Pablo Forner, los cuales se encargaron en sendos trabajos de resaltar las glorias de España. A ellos se sumó una incontable multitud de pequeños opúsculos y artículos de prensa escritos con este mismo objeto ${ }^{4}$. La propia Academia Española tomó cartas en el asunto en 1785 convocando un concurso de Oratoria con el tema siguiente:

«Una apología o defensa de la Nación, ciñéndose solamente a sus progresos en las ciencias y artes, por ser esta parte en la que con más particularidad y empeño han intentado obscurecer su gloria algunos escritores extranjeros, que llevados de sus engañosas preocupaciones y faltos de seguras noticias, han publicado obras llenas de injurias e imposturas».

No es este el lugar de seguir punto por punto esta apasionante polémica. Unicamente resulta necesario indicar que la historia del arte se instrumentalizó como un mito cultural de primera magnitud y que sus cumbres más universales fueron alanceadas para demostrar la ignorancia de los críticos de la nación española ${ }^{5}$. Así, por ejemplo, la defensa de Antonio Cavanilles permite hacerse una idea del papel otorgado a la reivindicación artística en la defensa de España: de los veintidos capítulos en los que divide dicha obra, nada menos que cuatro están dedicados a la exposición pública de la envidiable

4 Como el titulado "Continuación de la defensa de Cádiz, contra la descripción poco exacta de aquella ciudad hace Mr. Mason de Morvillers, en el tomo primero de Geografia moderna de la Encyclopedia metódica Francesan, publicado en el Memorial Literario, diciembre de 1787, Vol. XII, págs. 625 y 626. Alli, su autor afirma: «Sigue Mr. Mason su narrativa diciendo que la Catedral es antigua y pequeña (...) es cosa de admirar que después de 50 años que ha que se está labrando una hermosa y suntuosa Iglesia Catedral á expensas de este comercio y á imitación de la de San Pablo de Londres no haya llegado a noticia del Geografo moderno».

5 Algo parecido puede afirmarse de la historia de la literatura cuyo paralelo con la historia del arte en este tema es absolutamente innegable. Sobre este tema resulta útil la consulta de GLENDINNING, N., Historia de la literatura española, Barcelona, Labor, 1974, págs. 48-53. 
tradición en el terreno de las artes plásticas (Bellas Artes, Arquitectura, Pintura y Grabado). Además, en una corrección posterior (pág. 111, nota 2), añade la Escultura, que no había incluido antes por haberse «escrito estas Observaciones con alguna precipitación" ${ }^{\text {. }}$

En términos generales puede afirmarse que la herida que causó a los espanoles este autor debió ser grande y dolorosa, hasta el punto que su réplica se convirtió en un lugar frecuentado por distintos aficionados, como es el caso de Antonio Roca y Pertusa, académico de honor de la valenciana de San Carlos que, para demostrar la relevancia de este centro, afecta una actitud de retórico escándalo con esta pregunta:

"¿Qué es esto? ¿acaso nosotros, que por el amor de la verdad nos indignamos contra aquella pregunta del Extrangero ¿Qué debe Europa á España? ignorarémos, $\sigma$ afectarémos ignorar, quánto debe Valencia á su Real Academia de las tres Nobles Artes? A fe mía, que no a de ser asín ?.

En fin, los innumerables panegiristas de la patria que surgieron por doquier, introdujeron la cultura de forma genérica y las actividades artísticas especificamente, como un elemento fundamental en la reivindicación del honor de la nación española. Autores como José Vargas y Ponce, el cual, en su Apología de la Literatura Española, aborda la misión expresada en el título con ejemplos de diversa índole, de los cuales, los relativos a la pintura - no podía ser de otra forma - se refieren a la de los siglos xvi y xvn, calificando, entre otros, a Velázquez, como «aquel talento original desdeñador de todas las escuelas» o a Pablo de Céspedes como «el Corregio de la Andalucía» ${ }^{8}$. En este

\footnotetext{
6 A propósito de la pintura, afirma; «Esparia, que en otros tiempos produxo á Velázquez, Muri1lo, Ribera, Ribalta y otros muchos Pintores admirables, á pesar de la posesión de los mejores originales, de las escuelas famosas, ha visto sepultarse todas las bellas artes: por último ha dispertado (sic) de este largo letargo y admira hoy los talentos de Vergara, Bayeux, Maella \& C. Nosotros hemos visto á estos célebres artistas perfeccionarse con el estudio de la naturaleza, de los grandes modelos, y sobre todo por las lecciones vivas del famoso Mengs, el Rafael moderno, Pintor admirable que ha adornado con sus producciones el Palacio de nuestros Reyes y las casas particulares. Los que desearen conocer el fondo de riquezas que la España posee pueden leer la obra de D. Antonio Pons, Secretario perpetuo de la Academia de las Bellas Artes. El patriotismo de este excelente ciudadano no le impide el juzgar con la mayor imparcialidad y crítica más segura todas las obras de sus compatriotas). CABANILLE, Antonio, (sic), Observaciones sobre el articulo España de la Nueva Encyclopedia. Escritas en Francés por el Doctor $D$. Presbitero. $Y$ traducidas al Castellano por Don Mariano Rivera, en Madrid, en la Imprenta Real, MDCCLXXXIV [1784], págs. 23 y 24.

7 Contimuación de las Actas de la Real Academia de las Nobles Artes establecida en Valencia con el título de San Carlos, y relación de los Premios que distribuyó en 6. de Agosto de 1792. En Valencia, en la Oficina de D. Benito Monfort, Año 1792, pág. 29.

8 El título completo es Apología de la Literatura Española en las ciencias y en las Bellas Artes. Presentada a la Real Academia. Año 1785. Manuscrito depositado en el Archivo de la Real Academia de la Historia, sig. Papeles de Vargas Ponce, 51 (9-22-5-4224), sin paginar. En esta misma obra afirma: «Corregio al ver un quadro de Rafael exclamó: Yo también soy pintor. Permitaseme a la vista de tanto gran cuadro en que veo delineada la defensa de mi causa exclamar con entusiasmo: Yo también emprendo tu Apología, Españan.
}

Hispania, LVI/, núm. 192 (1996) 51-62 
mismo sentido, el célebre Leandro Fernández de Moratín, buen conocedor de cuestiones artísticas, escribió a Ceán Bermúdez comparando su Descripción artistica de la catedral de Sevilla con los tomos publicados por Carlos III sobre las excavaciones de Herculano, esto es, igualando en importancia la antigüedad clásica con el arte medieval peninsular ${ }^{9}$.

Personajes de la importancia de Antonio Ponz terciaron en la polémica Masson ${ }^{10}$. Por otra parte, esta circunstancia no debe extrañar en absoluto en un autor que ya había justificado la redacción del célebre Viage de España, como una defensa contra la obra Lettere d'un vago italiano ad un suo amico, de Norberto Caimo, que consideraba cargada de juicios injustamente descalificantes contra la nación española y sus habitantes. En esta misma línea resulta consecuente, casi necesario, que en su Viage fuera de España " cie igualmente esta intención de corte nacionalista, dedicando íntegramente el Prólogo del tomo II a la crítica de la Enciclopedia Metódica ${ }^{12}$, en unos términos que sorprenden un tanto por su dureza. De hecho, la apología de la patria constituye uno de los elementos justificatorios de toda la producción literaria de Antonio Ponz. Así, por ejemplo, manifestó hasta qué punto era sensible hacia las críticas de los extranjeros en su comentario a la obra de Edward Clarke, Letters concerning the Spanish Nation: Written at Madrid during the years 1760 and $1761^{13}$, cuya visión de las artes españolas era cier-

${ }^{9}$ Apéndice a la Descripción artística de la Catedral de Sevilla, Sevilla, en casa de la Viuda de Hidalgo y sobrino, 1805, pág. XXXI.

10 En términos generales puede encontrarse bastante información sobre viajeros extranjeros en RIBBaNs, Geoffrey, «Spanish national pride and foreign travelers in the eighteenth century», Dieciocho, vol. X, núm. 1, 1987, págs. 3-12.

11 por $D$. Secretario de la Real Academia de San Fernando, Madrid, Joachin Ibarra, MDCCLXXXV [1785], 2 vols. Se llevó a cabo una reseña en el Memorial Literario, t. VII, 1786, págs. 526 y 527 . Se realizó una segunda edición en Madrid, por la Viuda de Ibarra, MDCCXCI [1791], 2 vols. Se llevó a cabo, así mismo, una traducción al italiano Viaggio fuori di Spagna di D. Antonio Ponz Segretario della Reale Accademia di S. Fernando, etc. in cui si da notizia delle cose piú riguardevoli spezialmente intorno alle Belle Arti di Francia, d'Inghilterra e d'Olanda. Traduzione dall'originale Spagnuolo nell'idioma italiano. T. I. In Ferrara MDCCXCIV [1794]. Degli eredi di Giuseppe Rinaldi con approvazione. PUENTE, Joaquín de la, La visión de la realidad española en los viajes de don Antonio Ponz, Madrid, Editorial Moneda y Crédito, 1968, págs. 245-281, transcribe integramente el texto de Ponz.

12 Aunque, de forma un tanto sorprendente, entre los múltiples temas que aborda, no se encuentra el de las artes. Sin embargo, conviene aclarar que el autor de esta apología no fue el propio Ponz, sino un anónimo comunicante, cuyas opiniones fueron también parcialmente publicadas por BouRGoING, J. F., Tableau de l'Espagne moderne, Paris, 1807, t. I, pág. 308. Existe, así mismo, una copia manuscrita incompleta de este ataque anónimo a Masson en la Biblioteca Nacional de Madrid, sig. ms. 18565/4. Sobre todo ello vid. F. LóPEZ, op. cit., págs. 360-361 y nota 81. Las opiniones de Ponz a este respecto fueron muy apreciadas, como lo pone de manifiesto el artículo dedicado a este personaje por SAMPERE y GUARINOS, Juan, en su Ensayo de una biblioteca española de los mejores escritores del Reynado de Carlos III, En Madrid, en la Imprenta Real, MDCCLXXXVII [1787], págs. 254-259.

13 Londres: Printed for T. Becket and P.A. De Hondt, at Tully's Head in the Strand, MDCCLXIII [1763]. 
tamente epidérmica. «Perdoneme el señor Clarke - así comienza su alegato el viajero español-, que en materia de bellas artes es un ignorantón de primer orden». Después de descalificar las críticas vertidas hacia El Escorial asunto éste absolutamente innegociable por parte de la crítica española de la época ${ }^{14}$ - pasa revista al tema que realmente nos interesa dentro de la argumentación que pretendemos seguir: la defensa de la pintura barroca española; de la que Clarke, evidentemente, no sabía demasiado, ganando por ello las críticas de un Ponz sensiblemente molesto:

"Lo que admira es que se ponga a hablar de bellas artes uno que se da a conocer tan inteligente en ellas como un mozo de cordel. ¿quién ignora que el Espafroleto murió en Nápoles, donde hizo sus principales obras y donde se trasladaron a España y a muchas galerías de Europa? ¿Cuando ni en qué parte pintó techos o bóvedas Velázquez? ¿Ni quien, sino el doctor Clarke, ha dicho que el cuadro de los hijos de Jacob es de Murillo? ${ }^{15}$.

En otro momento arremete contra los ingleses de forma general:

En España todo se puede ver sin obstáculos y sin las socalifias que a cada paso experimenta el extranjero en Inglaterra, donde nada ve ni nada le enseñan si no paga, habiendo llegado la ruindad a poner tasa para enseñarle las cosas que encierran los palacios e iglesias. Tres o cuatro veces estafan a los curiosos que quieren ver la Torre de Londres, o bien a los que quieren ver por partes su decantada iglesia de San Pablo (...). ¿En qué parte de España sucede esto, ni en Madrid, ni en los lugares más infelices? ${ }^{16}$

En la misma obra, el propio Ponz se revolvió contra otros críticos que habían dañado el orgullo de los historiadores españoles dedicados a glosar los momentos cumbre de las artes nacionales. El más incisivo había resultado ser André Felibien (1619-1695), el cual en sus Entretiens sur la vie et les ouvrages des plus excellents peintres, concretamente en el "Neuvième Entretien», maltrató la figura de Velázquez, circunstancia doblemente grave al comparar el entusiamo manifestado por otros pintores como Rubens o Rembrandt ${ }^{17}$. La crítica a este autor - al que Ponz se refiere como Filibien-, se producía por haber demostrado su desconocimiento de los temas nacionales, al menospreciar la producción pictórica de lo que el francés consideraba los mejores pintores españoles: Cleante y Velasque, además de Ribera, fuera de los cuales

14 El marqués de Ureña afirma a propósito de este tema: «Se dice que los Montaf́eses, Berruquetes y Junis fueron ballenatos (...) todavia se disputa a Herrera la gloria de arquitecto del Escorial». El viaje europeo del marqués de Ureña (1787-1877), Cádiz, Unicaja, 1992. Sobre este tema ha tratado BoTTINEAU, Y., El arte cortesano en la España de Felipe V, págs. 248 y 249.

15 Viaje fuera de España, t. primero, Prólogo, págs. 1665b y 1666a de la edición de Aguilar de Madrid, 1947.

16 Ibidem, págs. 1664 b y $1665 \mathrm{a}$.

17 Comentario que contrasta con el de Mario Boschini (1613-1678), defensor a ultranza de la pintura veneciana, de la obra de Rubens y de Velázquez.

Hispania, LVI/, núm. 192 (1996) 51-62 
nada reconoce ${ }^{18}$. Sin duda, mucho más de lo que Antonio Ponz podía aceptar. Por ello afirma:

¿No quedamos lucidos y no quedará bien instruida Francia con este pasaje, en cuanto a noticias de pintores españoles? El citado autor no debía haber tocado este punto, o debiera haberle instruido siquiera superficialmente antes de tocarlo.

Porque, efectivamente, el tal Cleante es un "pintor que nadie ha conocido en España», a no ser que se refiera a Collantes o Escalante, que no son pintores de primera fila; pero, ignorar la figura de Velázquez es algo que sobrepasa los límites de lo tolerable:

Me mantengo en la opinión de que a muchos escritores franceses les son menos conocidas las cosas que suceden a la puerta de su casa, cual se puede considerar España, que las que suceden en la China. Habiendo tenido nuestra nación tantos pintores de mérito, anteriores al Cleante y Velasque de Filibien, en estos incógnitos, como él dice, empiezan y acaban las noticias pictóricas de España, con el fallo de que tal pais no ha dado grandes pintores ${ }^{19}$.

Todos estos comentarios demuestran que la producción de literatura artística de finales de siglo se mostró muy sensible ante el tema de la defensa de la historia de la pintura española, frente a las numerosas críticas que se llevaron a cabo en publicaciones extranjeras, preferentemente francesas, sobre todo a partir de la década de 1790 , en que las relaciones entre ambas naciones se vuelven especialmente turbias. Un personaje de importancia en lo que se refiere al pensamiento artístico español de finales de siglo, Diego Antonio Rejón de Silva, justificó así mismo la publicación de su obra La pintura. Poema didáctico en tres cantos alegando motivos patrióticos y, de la misma manera que Ponz, fue muy crítico con posturas como la del mencionado Félibien $-\mathrm{a}$ quien atribuye intenciones inconfesables ${ }^{20}-\mathrm{y}$ su desconocimiento de la historia de las artes en España:

18 "CleANTE \& Velasque étoient deux Peintres Espagnols contemporains du Cortone. Il y a dans le Cabinet du Roi un Païsage accompagné de figures, fait par Cleante; \& dans les apartemens bas du Louvre, plusieurs Portraits de la Maison d'Attriche peints par Velasque.

Que trouvez-vous, dit Pymandre, d'excellent dans les ouvrages de ces deux inconnus? Car je ne me souviens pas d'en avoir oüi parler: aussi n'est-il guéres sorti de grands Peintres de leur païs.

J'y remarque, lui répondis-je, les mêmes qualitez qui se rencontrent dans les autres qui n'ont pas tenu le premier rang, hormis qu'il semble à voir la maniere de ces deux Espagnols, qu'ils ayent choisi \& regardé la nature d'une façon toute particuliere, ne donnant point à leurs tableaux outre la naturelle ressemblance, ce bel air que releve \& fait paroître avec grace ceux des autres Peintres dont nous avons parlé». FÉLIBIEN, André, Entretiens sur les vies et sur les ouvrages des plus excellens Peintres anciens et modernes; avec la vie des architectes par Monsieur , A Trevoux, de l'Imprimerie de S.A.S., M.D.CC.XX.V. [1725], págs. 175 y 176.

19 Ibidem, t. I, carta IV, núm. 68, Ed. de Aguilar, pág. 1709b.

20 En Segovia: Por Don Antonio Espinosa de los Monteros, Año de 1786, págs. 103 y 104: «No parece posible que un aficionado tan inteligente, como Felibien, pierda todo su conocimiento en la Pintura al mirar las obras de un Español. Mas facil es atribuir este efecto á otra causa, de cuyo contagio debe estar libre todo hombre sábio, y en especial todo Historiadon. 
La Nación Francesa - afirma - tal vez extrañará el elogio que aquí se hace de nuestros Pintores; pero mas extrañamos nosotros el silencio que observan por lo común todas las obras suyas que tratan de las Bellas Artes á cerca de nuestros Profesores; y el tono decisivo con que se dice en algunas que no ha habido en España Pintor digno de fama ${ }^{2 !}$.

Rejón fue también muy crítico con uno de los grandes autores de principios de siglo, concretamente con el Abate Du Bos (1670-1742), el cual se había pronunciado en términos igualmente descalificantes en contra de la tradición pictórica peninsular. Afirma el autor francés en la traducción de Rejón de Silva:

Aunque los Españoles han tenido algunos Soberanos de tanta magnificencia, y de tanto amor á la Pintura, qual jamás ha mostrado alguno de los Papas; sin embargo una Nacion tan fertil como ésta en Personages grandes y aun en excelentes Poëtas (...), no ha producido Pintor alguno de primera clase; y aun de segunda apenas se encuentran dos 22 .

La defensa establecida por el autor español se basó en citar listas muy completas de pintores nacionales que pusieran de manifiesto la escasa credibilidad de las opiniones vertidas por el teórico francés.

En otro orden de cosas, resulta interesante poner de manifiesto que este autor español se mostró también muy sensible ante otro de los grandes mitos de la centuria, de enorme importancia en el terreno de la rivalidad entre las distintas naciones: se trata del llamado determinismo climático, del que Winckemann había escrito abundantemente. En función de esta idea, desarrollada originariamente por Montesquieu, el clima constituía un elemento fundamental en la configuración de lo que podíamos calificar como el carácter de las naciones, esto es, aquellas características propias de un pueblo que permitían distinguirlo de los demás. Tradicionalmente este argumento había demostrado la preeminencia de la Europa Mediterránea - ahí están los ejemplos de Grecia y Roma para demostrarlo- aunque, en opinión de Du Bos las cosas habían empezado a cambiar:

No solo en los Países frios ó humedos con exceso dexa de florecer las Artes, que también en algunos que son templados se hallan decaídas.

Se refiere, naturalmente, a España. La contestación implícita a esta argumentación no se llevó a cabo en esta obra, sino en la Notas ó ilustraciones del Traductor Español que preceden su traducción manuscrita de la Historia de las Artes, entre los antiguos ${ }^{23}$, de Winckelmann, en las que reconoce una cierta prelación al norte de Europa en lo que se refiere a las matemáticas o la filosofía

21 lbidem, pág. 98.

22 Ibidem, pág. 99. Rejón cita la cuarta edición de las Réflexions critiques sur la poésie et la peinture, Paris, 1755, t. II, pág. 162.

${ }_{23}$ Por $M r$ Presidente de Antiguedades en Roma, individuo de la $R^{1}$. Sociedad de antiguedades de Londres, de la Academia de Pintura de S. Lucas, y de la Etrusca de Cortona, obra traducida

Hispania, LVU, núm. 192 (1996) 51-62 
(Newton, Leibniz); aunque, eso sí, no hay duda alguna de que los paises del sur están más capacitados para todo tipo de manifestación artística, como lo demuestran ciertos casos:

Petrarca, Garcilaso, Herrera, Rafael, Miguel Angel, Rivera, Velázquez, etc. porqe para la Poesia y Bellas Artes es menester principalmente un gran fuego de imaginación ${ }^{24}$.

En fin, podrían ser muchos más los autores que pusieron sus plumas al servicio de la defensa de la patria y para combatir los nocivos efectos de la critica foránea. Lo más interesante es que estas críticas tuvieron el efecto de un espejo en el que los españoles tuvieron la necesidad de mirarse y, más importante, de definirse como pueblo. En este ejercicio de autoanálisis se llevaron a cabo interesantísimas reflexiones sobre otro de los termas estrella de la época: el conocimiento de la historia y la necesidad de reconocer sus momentos estelares, con los que construir un acervo común con el que todos los miembros de un colectivo nacional pudieran identificarse y mostrar su orgullo por la pertenencia a una nación en concreto. Y, de nuevo, podríamos identificar a los mayores talentos de la centuria empeñados en esta empresa común. Personajes como Jovellanos, para el que la figura de Velázquez aparece aumentada con la dimensión de un gigante frente al que ni los franceses, ni ningún otro pueblo europeo podía proponer ejemplos equiparables. Este nuevo "principe de los pintores españoles" ${ }^{25}$, aparece a los ojos de la época como un auténtico héroe que, frente a las provocaciones externas, había sostenido "en quanto estaba de (su) parte nuestra reputacion vacilante" ${ }^{26}$. El

del alemán al francés, y de éste al Castellano, pr. d. D.A.R.D.S. afio. 1784. Se conserva manuscrito en el Archivo de la Academia de San Fernando de Madrid (A.A.S.F.), sig. ms. 41-44. Afirma Rejón en la pág. 291v: «Los Paises en que ésta (la Naturaleza) se muestra mas bella, apacible, y benigna es en donde se hallan producciones de las Artes de imaginación $q^{e}$ propone y esparce $p^{r}$ todas partes la Naturaleza en unos climas templados. Así que en aq paises donde el aire spre. es puro y suave, el sol casi siempre resplandeciente con sus rayos, la tierra siempre amena, llena de arboles, plantas, flores, fuetecillas, y frutos (...) alli es donde lo que hacen, como están siempre rodeados de objetos hermosos y atractivos (...) no tienen idea sino de la dulzura y belleza de la naturaleza que los rodeay.

24 Ibidem, págs. $289 \mathrm{v}$ y 290 . Resulta enormemente interesante comprobar que este modelo de funcionamiento dejaba en un lugar francamente delicado a Mengs, de origen bohemio, asunto éste de importancia para una persona como Rejón, al que podría calificarse como «mengsiano crítico». Sin duda por ello, se vió en la obligación de explicar la excepción que suponía el caso de este artista: «Pero esta no es regla capaz de excepción, $p^{s}$ bien conoce el mundo a un Alemán Pintor de primer orden como d. Ant ${ }^{\circ}$ Rafael Mengs (...); sin $\mathrm{q}^{\mathrm{e}}$ la flema y frialdad saxona impidiese a la sabia imaginación de aquel inventar las bellisimas composiciones $\mathrm{q}^{\epsilon}$ admiran los inteligentes en el Real Palacio de Madrids. Págs. 290v y 291.

25 Distribución de los Premios concedidos por el Rey nuestro Señor a los discipulos de las tres Nobles Artes, hecha por la Real Academia de San Fernando en la Junta Pública, de 13 de Julio de 1796, Madrid, en la Imprenta de la Viuda de Ibarra, pág. 2.

${ }_{26}$ VARGAS PONCE, José, «Discurso histórico sobre el principio y progreso del Grabado», en Distribución de los premios concedidos por el Rey nuestro Señor a los discípulos de las Nobles Artes, hecha por la Real Academia de San Fernando en la Junta Pública de 4 de agosto de 1790, Madrid en la Imprenta de la Viuda de Ibarra, pág. 62. 
propio Isidoro Bosarte, en su obra, el «Discurso sobre la restauración de las artes en Españan, afirmó expresamente que sus palabras iban dirigidas a los extranjeros, por motivos patrióticos, para corregir determinados errores extendidos que hacían poca justicia del arte español ${ }^{27}$. En fin, Eugenio Llaguno y Amirola se mostró en todo momento muy respetuoso con la tradición española frente a las frecuentes críticas de su amigo José Nicolás de Azara. En una de sus cartas le manifestó su preferencia por la pintura española en estos términos:

Italianos y franceses dicen que no hemos tenido ninguno [se refiere a los pintores] de primer orden: será así, pero en los Salones de Palacio delante de Velázquez todos quedarán pegados a la pared ${ }^{28}$.

Lo cierto es que el nacionalismo apasionado se convirtió en un tema especialmente recurrente en los escritos de la época, crispando un tanto los ánimos de sus protagonistas, alguno de los cuales creyó descubrir en estas polémicas una cierta incapacidad para asumir determinados aspectos negativos, de lo que entonces se entendía como la manera de ser de los españoles ${ }^{29}$. Ahora bien, ¿Cuales eran los argumentos artísticos que permitían sentir un legítimo orgullo por pertenecer a esta nación maltratada por los extranjeros?

${ }^{27}$ Gabinete de lectura española, o colección de muchos papeles curiosos de escritores antiguos y modernos de la Nación, núm. II, Prólogo y págs. 40-45.

${ }^{28}$ Y continúa: «Murillo fué el Corezo [Correggio] de España, quando quiso serlo. Ni uno ni otro son conocidos en Roma: solo en Inglaterra se estiman y pagan como merecen. Joanes el Valenciano es de la casta de Rafael: Carreño, Pereda, Cerezo, Claudio Coello se las empatan en el colorido a Ticiano, y tienen otras muchas virtudes, Rivalta fue grande hombre. De Alonso Cano hay cosas que parecen vivas y que handan fuera del lienzo. Nada digo de algunos Cordoveses y Sevillanos por que no los he visto.» SALAS, Xavier de, "Cuatro cartas de Azara a Llaguno, y una respuesta de éste», Revista de Ideas Estéticas, t. IV, 1946, pág. 103.

29 Alguno de ellos tan próximo a la historia de las bellas artes como Bernardo de Iriarte, viceprotector de la Academia de Madrid y gran conocedor del arte del periodo, del cual se conserva esta opinión: «Rompiose y manifestose esta especie de guerra nacional tenaz y ridiculamente con ocasión de haber Mr. Masson compuesto y estampado en la Nueva Enciclopedia el artículo Espagne, y se trabajó con encono en promover é incitar el odio de nacion á nacion protegiendo y fomentando los escritos apologéticos que entonces salieron, en que tanto se disparató con daffo, atraso, necia presunción, jactancia é ignorancia, que se procuró aumentar entre los españoles, con sentimiento y dolor de los hombres de juicio é instrucción. Llovieron apologistas con motivo de aquel fatal artículo. El primero y principal de ellos fue Cavanilles, que publicó en Paris su obra en Francés, y sobre los errores y ridiculeces que contiene, como sobre las malas resultas, formé un extracto y crítica, que pasé á manos del propio Conde de Floridablanca por insinuación suya. No obstante los convencimientos de este escrito, que dejó confuso e intimamente persuadido al Ministro, ó él por si, llevando adelante su sistema de aversión á los franceses y el que ya había adoptado de ponderar y figurar el ventajoso estado de la Espaffa en todas líneas bajo su ministerio, ó estimulado y lisonjeado de los que le rodeaban, empleó la pluma mordaz e indiscreta del difamador público D. Juan Pablo Forner (alias Segarra), para que éste compusiese, como compuso, una voluminosa, impertinente y fastidiosa Apología, que se imprimió á Reales expensas en la Imprenta Real.» cit. por CoTARELO Y MORI, Emilio, Iriarte y su época, Madrid, Sucesores de Rivadeneyra, 1897, págs. 315 y 316.

Hispania, LVI/, núm. 192 (1996) 51-62 
¿Cuales los mitos que se propusieron para combatir las críticas provenientes del exterior? La respuesta es una sóla: aquello que podía esgrimirse como específicamente hispano, aquello que permitiera a los españoles singularizarse como pueblo y que les autorizaba para sentir orgullo por la pertenencia a esta nación y que, incluso, les permitiera hablar de una cierta preeminencia histórica de lo nacional frente a las producciones foráneas, sean culturales, científicas, industriales, comerciales, militares o de cualquier otro ámbito. En definitiva, en el terreno estrictamente cultural la apología se desarrolló con la exaltación de los logros de los dos siglos de oro: el xvI y xvi, fundamentalmente de este último; de aquello, en definitiva, que era definido con evidente orgullo como «el genio y carácter español " ${ }^{30}$ o "el genio del pais» ${ }^{31}$.

Este asunto constituye una de las señas de identidad de las últimas décadas del siglo, que rebasa los límites de lo propiamente cultural. Por otra parte y como puede imaginarse, las bellas artes no fueron más que una parcela de la cultura en la que se puede observar este fenómeno. Un ejemplo muy interesante es el de X. Lampillas, en cual, en su Ensayo histórico-apologético de la literatura española ${ }^{32}$, lleva a cabo un análisis de los procedimientos literarios para acercarnos a distintos periodos históricos. A propósito de los límites del clasicismo y del naturalismo, afirma en un determinado momento:

Pero hablemos filosóficamente. Hay costumbres que llevan impreso el carácter propio de la naturaleza, y por consiguiente son comunes a todas las naciones y a todos los siglos; tales son aquellas inclinaciones que acompañan las varias edades del hombre, y que pinta elegantemente Horacio en su poética; tales pueden llamarse también aquellos caracteres que distinguen los vicios: por ejemplo el carácter del avaro, del colérico, del soberbio, etc. Otras costumbres menos generales forman, según su diversidad, el diferente carácter de las naciones; finalmente, otras llevan el distinto sello de los siglos, que varían conforme a los usos en las sociedades civiles, Y Continúa a continuación: «En este supuesto digo que para juzgar con acierto del mérito de un autor en la descripción de los caracteres, es necesario observar si retrata los impresos por la naturaleza, y si convienen con ésta; o si pinta los de alguna nación particular, o las costumbres de cierto tiempo, entonces es indispensable trasladarse a aquella nación que describe el autor, $o$ al tiempo en que escribió, para juzgar después si los retratos son conforme a los originales ${ }^{33}$.

Esta forma de pensar pone de manifiesto que el problema del nacionalismo tuvo en los últimos años del siglo xVII amplias y profundas derivaciones. Por

30 Cean Bermúdez, Juan Agustín, Historia del arte de la Pintura. Por D. , t. VI, 1824, pág. 1. Se conserva manuscrita en el A.A.S.F., sig. Ms. 44-45.

31 Ponz, Antonio, Viage de España, t. IX, carta última, núm. 13. Por una errata inexplicable, las palabras «del pais» fueron omitida en la edición preparada por la editorial Aguilar.

${ }^{32}$ Cit. por LópEZ, François, Juan Pablo Forner..., pág. 336.

33 Otro muchos ejemplos en este mismo sentido, referidos al teatro y la novela aparecen en ÁlVAREz BARRIENTOS, Joaquín, «Del pasado al presente. Sobre el cambio del concepto de imitación en el siglo XVII español», Nueva revista de filologia hispánica, t. XXXVII, 1990, núm. 1, págs. 224 y 225.

Hispaniz, LVII, núm. 192 (1996) 51-62 
una parte, esta mirada al pasado al mismo tiempo nostálgica y reivindicativa, provocó la necesidad de "crear" una historia del arte moderna, hasta ese momento inexistente, que facilitase el conocimiento del pasado con unas garantías que podíamos calificar como cientificas, no susceptibles, por tanto, de manipulaciones intencionadas. De ninguna manera puede considerarse una casualidad que en esta época se produjera el nacimiento de la moderna historia del arte, cuyas obras continúan en buena medida siendo útiles para los modernos estudios. Por otra parte, existen numerosas evidencias de las dificultades con las que se enfrentaron los partidarios de la vigencia de la estética clásica para exponer públicamente sus convicciones artísticas en un ambiente no especialmente propicio, en el que, la afiliación a un sistema de pensamiento supranacional, podría valerles enojosas descalificaciones de renegados o malos patriotas. De hecho, en la época indicada se produjo un auténtico debate, todavía mal conocido por los historiadores, sobre la preeminencia de la pintura barroca o la de corte clasicista, con un trasfondo indudablemente patrítico, que comenzó a cuestionar la vigencia de los mitos clásicos mucho antes de que las propuestas románticas debilitasen a Zeuxis frente a Velázquez. 